\title{
Beam-Foil-Excited Auger Transitions in Neon *
}

\author{
K.O. Groeneveld, R. Mann ${ }^{\star \star}$, G. Nolte, S. Schumann, R. Spohr $\star \star$ \\ Institut für Kernphysik der Universität Frankfurt (Main), Germany

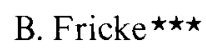 \\ Gesamthochschule Kassel, Germany
}

Received June 4, accepted June 18, 1975

\begin{abstract}
Energy spectra of electrons ejected from collisions between a carbon foil and $\mathrm{Ne}$ projectiles with energies between 1.4 and $20 \mathrm{MeV}$ have been measured. Continuous and discrete electron energy distributions are observed. Auger transitions of foil-excited $\mathrm{Ne}$ have been studied. Using relativistic Dirac-Fock multiconfiguration calculations, most of the measured Auger transitions have been identified.
\end{abstract}

\section{Introduction}

Excited states in Neon decaying via Auger electron emission have been investigated by a number of excitation modes [1]. Beam foil spectroscopy has become a powerful tool [2] in the study of excited atomic states of various ionization states. In this paper results are presented from Auger electron transitions from states excited with the beam foil technique.

\section{Experimental Set Up}

The Van de Graaff facilities at the University of Frankfurt (Main) have been used to produce Ne beams between 1.4 and $20 \mathrm{MeV}$ energy. With a coaxial cylinder electron spectrometer (described elsewhere [3]), energy spectra of electrons ejected at $\beta=42.3^{\circ}$ with respect to the beam direction and with respect to the spectrometer axis from foil excited projectiles were measured. Targets of carbon foil, sufficiently thin (about $10 \mu \mathrm{g} / \mathrm{cm}^{2}$ ) to provide for minimum straggling effects of the beam in the foil $[4,5]$, were used to excite the electronic states of the projectiles. The measured electron energy resolution at FWHM is $0.7 \%$.

\footnotetext{
* Work supported by the Bundesministerium für Forschung und Technologie, Germany.

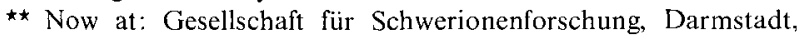
Germany.

$\star \star \star$ Work supported by Gesellschaft für Schwerionenforschung, Darmstadt, Germany.
}

\section{Results of Continuous Specira}

Fig. 1 shows typical electron energy spectra of $\mathrm{Ne}$ incident on carbon at bombarding energies between 1.4 and $20 \mathrm{MeV}$.

$E_{\max }=\left(4\left(m_{e} / m_{\mathrm{Ne}}\right) E_{\mathrm{Ne}}\right) \cos ^{2} 42.3^{\circ}$

gives the maximum energy transfer from the projectile to an electron at rest. The sharp drop in electron intensity at $E_{\max }$ is clearly seen in the figure. Contributions of electrons with energies $E_{e}>E_{\max }$ to the continuous distribution are due to the non-zero binding energy of the ejected electrons.

\section{Results from Discrete Energy Spectra}

Auger transitions excited by the projectile in the target atoms are weak compared to the intensity of continuous electron contributions for $\beta=42.3^{\circ}$; at the highest studied incident energy the carbon KLL Auger transitions become barely visible. The energies of Auger electrons $E_{e}^{\text {c.m. }}$ emitted in flight from the foil excited Ne beam are shifted in the laboratory frame $\left(E_{e}^{\text {lab }}\right)$.

$$
\begin{aligned}
& E_{e}^{\text {c.m. }}=E_{e}^{\text {lab }}+E_{\mathrm{Ne}}\left(m_{e} / m_{\mathrm{Ne}}\right) \\
& -2 \cos \left(42.3^{\circ}\right)\left(E_{e}^{\mathrm{lab}}\left(m_{e} / m_{\mathrm{Ne}}\right) E_{\mathrm{Ne}}\right)^{1 / 2}
\end{aligned}
$$

where $m_{\mathrm{e}}, m_{\mathrm{Ne}}$ are the masses of the electron and $\mathrm{Ne}$ respectively, and $E_{\mathrm{Ne}}$ is the projectile energy. In Fig. 1 these Auger transitions emitted in flight from 


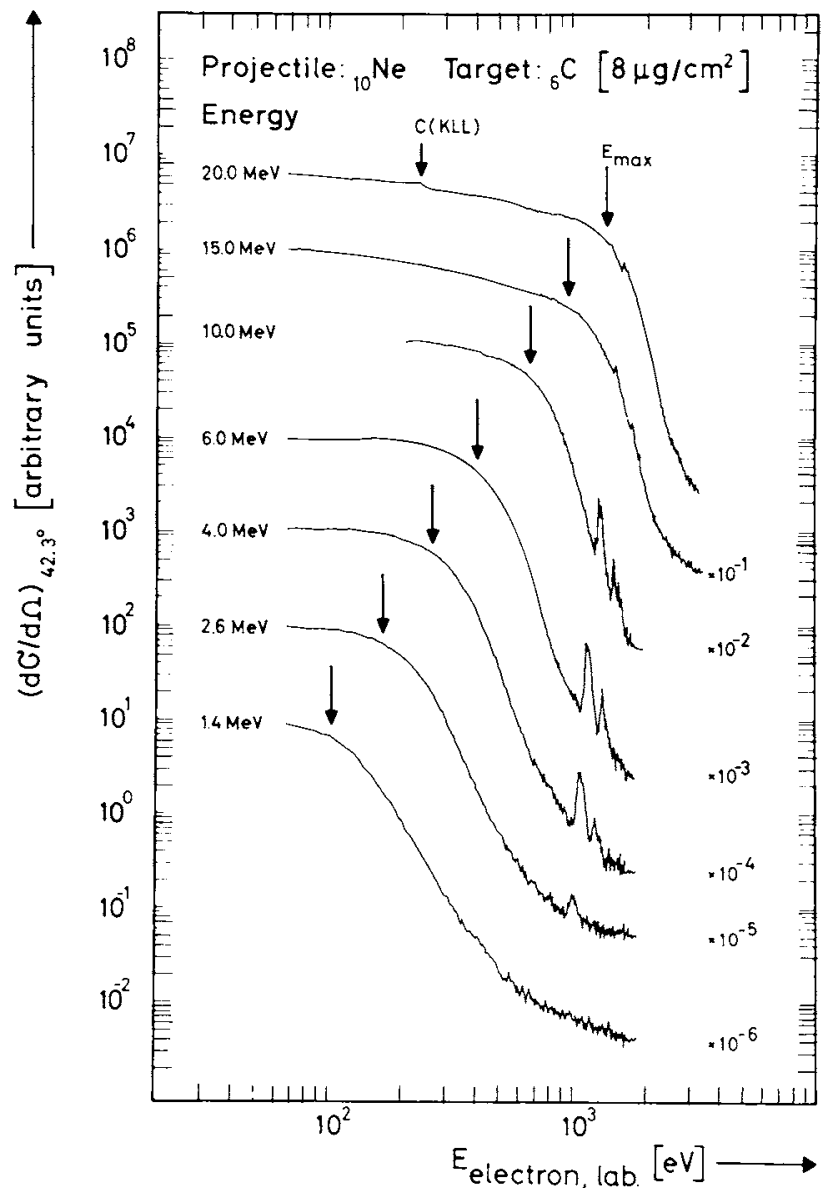

Fig. 1. Energy spectra of electrons emitted by bombarding solid carbon targets with Neon at different incident energies the projectiles are clearly seen between $E_{e}^{\mathrm{lab}}=1$ and $2 \mathrm{keV}$. They have the projectile energy dependence given by Eq. (2).

To study these transitions with a reasonable peak to background ratio, the projectile energy may not be too low since the ionization cross section then becomes too small (Fig. 1); on the other hand, the projectile energy may not be too high since the continuous background fills in under the Auger lines. In addition, at low projectile energies a broader velocity distribution of the projectiles introduced by energy and angular straggling in the exciting carbon foil contributes considerably to the measured electron line width.

For studying in detail projectile Auger transitions under the present experimental conditions, an optimum incident projectile energy between 6 and $10 \mathrm{MeV}$ can be chosen by considering the data in Fig. 1 . Since the projectiles penetrating through the solid foil undergo many successive collisions (typical time between two successive collisions is $10^{-15} \mathrm{sec}$ ), the ions emerging from the foil are in equilibrium excitation and equilibrium charge states; the incident charge state of the projectiles is irrelevant, i.e. the spectra are independent of the incident charge state for the foil thicknesses used in this experiment [6].

The average equilibrium charge state $\bar{q}$ of the projectiles after leaving the solid foil is predominantly determined by the incident beam energy e.g. $\bar{q}=6.7$ for Ne projectiles of $10 \mathrm{MeV}$ energy [7]. By proper choice of the projectile energy a particular charge state can be populated preferentially.

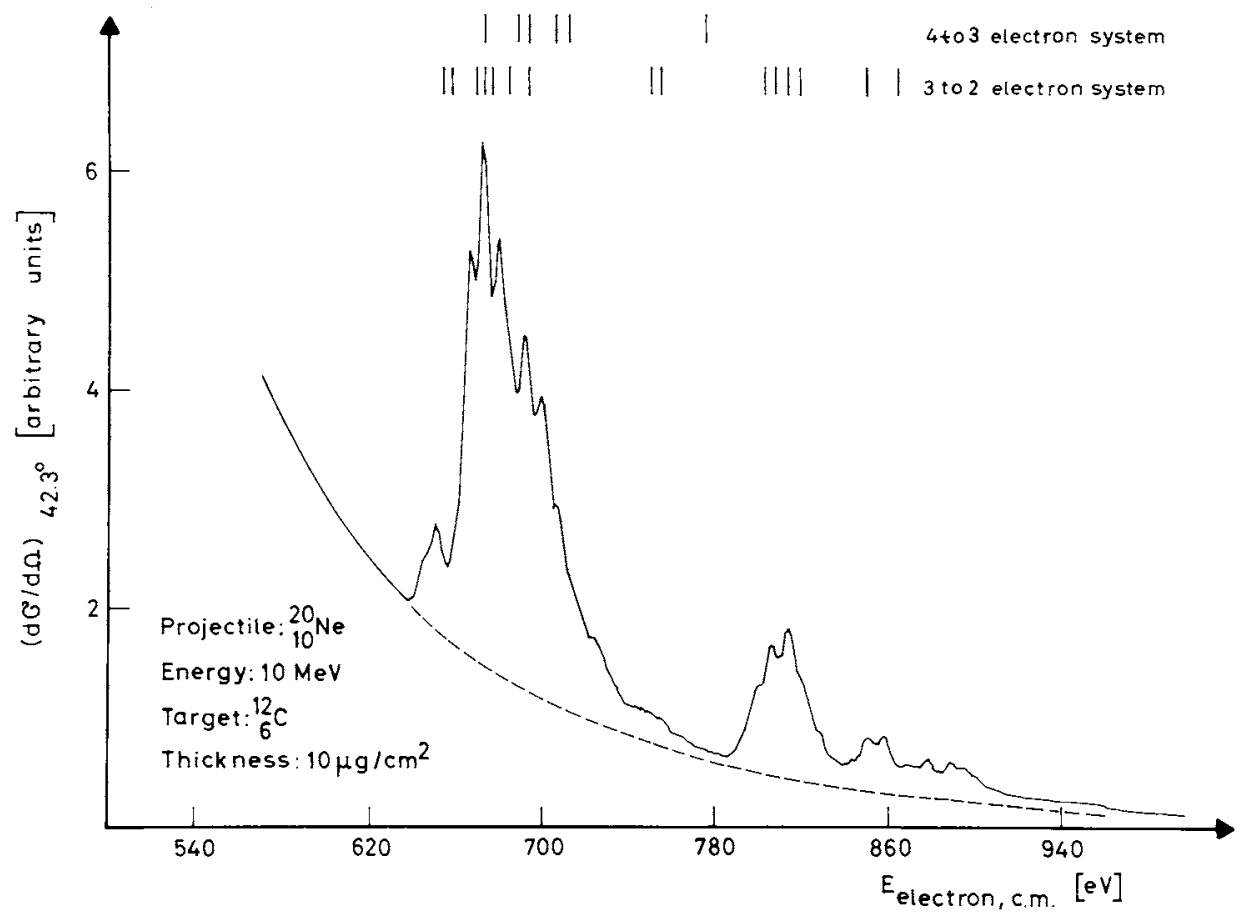

Fig. 2. Auger spectrum of foilexcited Neon. On top the calculated transition energies of the decay of the 4 and 3 electron systems are indicated (see Table 1) 
Table 1. Auger transitions in Neon

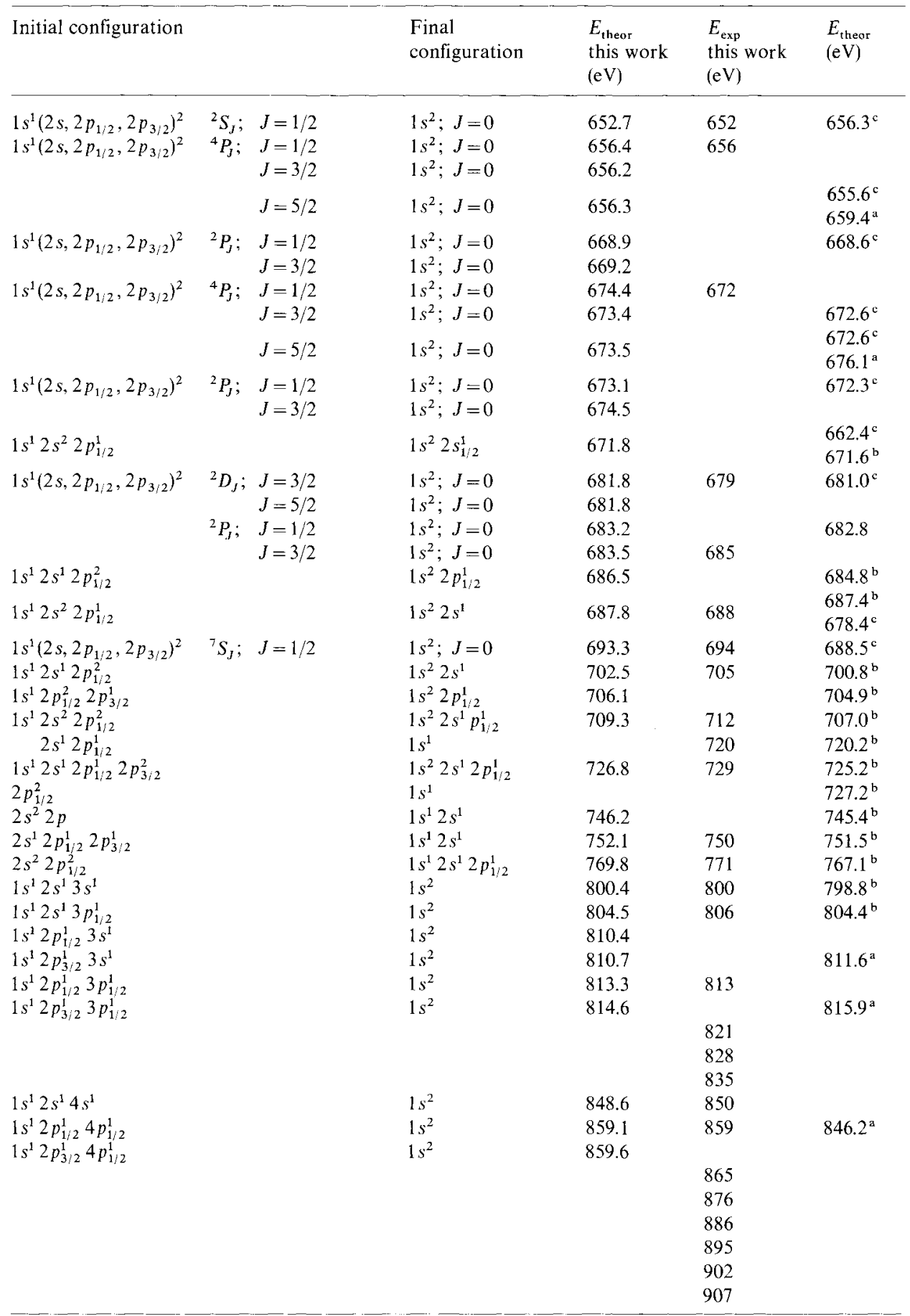

a Holøien, E., Geltmann, S.: Phys. Rev. 153, 81 (1967).

b Bhalla, C. P., Folland, N.O., Hein, M. A.: Phys. Rev. A 8, 649 (1973).

'Matthews, D. L., Johnson, B. M., Moore, C. F.: Atom. Data and Nucl. Data Tables 15, 40 (1975).

Fig. 2 shows in detail the Ne Auger spectrum obtained from the beam foil experiment at $10 \mathrm{MeV}$ projectile energy. The mean energy loss of the projectiles in the carbon target foil $\left(154.3 \mathrm{keV} / 10 \mu \mathrm{g} / \mathrm{cm}^{2}\right)$ [8] and the
Dopplershift (Eq. (2)) are taken into account. The background, indicated by the dashed curve, is interpolated between the continuous electron contribution above and below the Ne Auger transitions. The mea- 
sured lines (Table 1) may be attributed to transitions in $\mathrm{Ne}^{5+}, \mathrm{Ne}^{6+}, \mathrm{Ne}^{7+}$ or $\mathrm{Ne}^{8+}$ which are the preferably populated charge states for $10 \mathrm{MeV} \mathrm{Ne}$ in having passed through a solid foil [7].

\section{Theoretical Interpretation}

To get a first idea of the interpretation of the lines observed in the experimental spectra we performed relativistic Dirac-Fock-Slater calculations [9]. They already showed that the main groups in the spectra were transitions from 4 to 3 and 3 to 2 electron systems.

A more detailed interpretation was then done in the next step using relativistic Dirac-Fock calculations with one configuration only [10]. With this method several lines were reproduced with high accuracy, whereas some lines were still off by many eV. Because most of the lines in the first group between 640 and $700 \mathrm{eV}$ are transitions from a 3 electron configuration with two electrons in the $n=2$ states to the $1 s^{2}$ configuration, we extended the calculation to a full selfconsistent relativistic Dirac-Fock multiconfiguration calculation [10] taking into account all possible states which can be constructed with one electron in the $1 s$ state and two in the $2 s, 2 p_{1 / 2}$ and $2 p_{3 / 2}$ states.

This leads to 7 states with $J=1 / 2,6$ with $J=3 / 2$ and 3 with $J=5 / 2$ including both negative and positive parity. In the non-relativistic LS coupling nomenclature we call them ${ }^{4} P_{1 / 2,3 / 2,5 / 2},{ }^{2} P_{1 / 2,3 / 2}$ and ${ }^{2} P_{1 / 2,3 / 2}$ originating from the $1 s^{1} 2 s^{1} 2 p^{1}$ configuration, ${ }^{2} D_{3 / 2,5 / 2}$, ${ }^{4} P_{1 / 2,3 / 2,5 / 2},{ }^{2} P_{1 / 2,3 / 2}$ and ${ }^{2} S_{1 / 2}$ from the $1 s^{1} 2 p^{2}$ configuration and ${ }^{2} S_{1 / 2}$ from the $1 s^{1} 2 s^{2}$ configuration. The calculation consists of solving the total Hamiltonian of the system

$H=\sum_{i} H_{D}(i)+\sum_{i j}\left(r_{i j}^{-1}+H_{B}(i, j)\right)$,

where $H_{D}$ is the relativistic one-electron Hamiltonian, $r_{i j}^{-1}$ the Coulomb repulsion, and $H_{B}$ the Breit Hamiltonian operator which is taken into account by first order perturbation calculation. The total wavefunction for a bound state of the $N$ electron atomic system is assumed to be

$\Psi=\sum_{n=1}^{N} W_{n} \varphi\left(1,2, \ldots, N, J^{2}, J_{z}\right)$

where $\varphi$ is an eigenfunction of $J^{2}$ and $J_{z}$ and the sum goes over all configurations with $W_{n}$ the weight of each configuration.

The result of the calculations for our problem is given in Table 1 . To identify the states we use, as stated above, the non-relativistic nomenclature although our calculations are fully equivalent to intermediate cou- pling calculations. The experimental data agree very well with the absolute theoretical values. The two groups of lines at higher energy can best be interpretated as transitions between the analog states where the two outer electrons are in the $n=3$ respectively $n=4$ states, where the splitting pattern is very similar. The calculated transition energies are also shown in the Fig. 2.

\section{Conclusions}

By bombarding a thin carbon foil with $10 \mathrm{MeV} \mathrm{Ne}$ projectiles it is possible to get a sufficiently good peak-to-background ratio to measure discrete Augerlines of the ionised and excited $\mathrm{Ne}$ projectiles. For interpretation of the experimental results relativistic Dirac-Fock calculations were performed. Most of the observed lines could be identified as transitions from 4 to 3 or 3 to 2 electron states.

To obtain further information about these highly ionised and excited states a coincidence experiment and lifetime measurements by a time of flight method are under way.

\section{References}

1. Sevier, Kenneth D.: Low Energy Electron Spectrometry. New York: Wiley-Interscience 1972

2. Martinson, I., Gaupp, A.: Physics Reports 15C, 113 (1974) Burch, D., Stolterfoht, N., Schneider, D., Wieman, H., Riesley, J.S.: Phys. Rev. Lett. 32, 1151 (1974)

3. Dietz, E., Groeneveld, K.O., Spohr, R., Staudte, R.: Nucl. Instr. and Meth. 105, 467 (1972)

4. Groeneveld, K.O., Spahn, G.: Nucl. Instr. and Meth. 123, 425 (1975)

5. Schmidt-Böcking, H.: Private communication

6. Betz, H. D.: Rev. Mod. Phys. 44, 465 (1972)

7. Barette, L., Knystautas, E.J., Drouin, R.: Nucl. Instr. and Meth. 110, 29 (1973)

8. Northcliffe, L.C., Schilling, R.F.: Nucl. Data 7A, 253 (1970)

9. See for example: Liberman, D.A., Cromer, D.T., Waber, J.T.: Comp. Phys. Comm. 2, 107 (1971)

10. Desclaux, J. P.: Comp. Phys. Comm. 9, 31 (1975)

K.O. Groeneveld

R. Mann

G. Nolte

S. Schumann

R. Spohr

Institut für Kernphysik

der Universität Frankfurt (Main)

D-6000 Frankfurt (Main) 90

August-Euler-Straße 6

Federal Republic of Germany

B. Fricke

Gesamthochschule Kassel

Theoretische Physik

D-3500 Kassel

Heinrich-Plett-Straße 40

Federal Republic of Germany 